Preprint typeset in JHEP style. - HYPER VERSION

hep-th/0102016

\title{
Solitons in 2+1 Dimensional \\ Non-Commutative Maxwell Chern-Simons \\ Higgs Theories
}

\author{
Avinash Khare \\ Institute of Physics, Sachivalaya Marg, Bhubaneswar, 751005, India \\ E-mail: khare@iopb.res.in \\ M. B. Paranjape* \\ Departamento de Física Teórica, Facultad de Ciencias, Universidad de \\ Zaragoza, 50009, Zaragoza, España \\ E-mail: paranj@lps.umontreal.ca
}

\begin{abstract}
We find soliton solutions in the $2+1$ dimensional non-commutative Maxwell Chern-Simons Higgs theories. In the limit of the Chern-Simons coefficient going to zero, these solutions go over to the previously found solutions in the non-commutative Maxwell Higgs theories. The new solutions may have relevance in the theory of the fractional quantum Hall effect and possibly in string vacua corresponding to open strings terminating on D2 branes in the presence of D0 branes.
\end{abstract}

KEYWORDS: non-commutative gauge theories, solitons, quantum Hall effect.

Dedicated to... the pursuit of knowledge.

\footnotetext{
*permanent address: Groupe de physique des particules, Laboratoire René-J.-A.-Lévesque, Université de Montréal, C. P. 6128, succ. centre-ville, Montréal, Québec, Canada, H3C 3J7
} 


\section{Contents}

1. Introduction 1

2. Action and Equations of Motion and Comments 3

2.1 Action and Equations of motion 3

2.2 Comments on Non-Commutative Yang-Mills Theories, the Coefficient of the Chern-Simons Term and Gauge Invariance 3

3. Solutions 5

3.1 Previously found solutions 5

3.2 A new solution 6

3.3 Solutions with Chern-Simons term 6

3.3.1 Time dependent solutions 7

3.3.2 Static Gauge Ansatz 8

3.3.3 More General Time-Dependent Solution 10

3.4 New Solution at $e=0 \quad 10$

4. A Re-Formulation of the Non-Commutative Abelian Higgs Model 12

5. Conclusions 14

\section{Introduction}

In the last few years, the noncommutative field theories have attracted considerable attention. This is primarily because such theories appear naturally in the various limits of $\mathrm{M}$ theory, as limits of string theories with backgrounds of open strings terminating on D2 branes, and are also relevant to aspects of string and D-brane dynamics [1]-[3]. They also appear as theories describing the behaviour of a two dimensional electron gas in the presence of a strong, external magnetic field, the quantum Hall effect [⿶]. They exhibit an interesting space uncertainty relation [5]. Being non-local, understanding of these theories will help us in understanding the consequences of the breakdown of locality at short distances.

By now, several people have looked at both the perturbative and non-perturbative aspects of the non-commutative field theories. In particular, several people [7]-16], among many others, have looked at the soliton solutions in such theories. For example, Jatkar et. al. [17], Lozano et. al. [18] as well as Polychronakos [19] and Bak et. 
al. 16, 14 have looked at non-commutative $U(1)$ gauge theory with Higgs field and have obtained the vortex (or what Polychronakos calls as the flux tube) solutions. There is an important difference between the solutions obtained in 119, 16, 14 and the other two. In particular, whereas the solutions obtained in the former are singular in the $\theta \rightarrow 0$ limit ( $\theta$ being the non-commutative parameters defined below), the solutions obtained in the other two papers are nonsingular in this limit.

The purpose of this note is to look at the role of the Chern-Simons term on these flux tube solutions. In particular, we consider the non-commutative abelian Higgs model with Chern-Simons term in $2+1$ dimensions and obtain soliton solutions which represent flux tubes with both static electric and magnetic fields. It turns out that whereas our solutions are singular in the $\theta \rightarrow 0$ limit, they smoothly go over to the known solutions [19] in the limit of the Chern-Simons coefficient going to zero. Many authors have considered non-commutative gauge theories with a Chern-Simons term added [24. The specific case of self-dual solitons has been studied by Lozano et. al. [15].

Non-commutative gauge theories are described via two equivalent descriptions. Starting with the standard commutative gauge theory action and then re-interpreting any product of fields appearing in terms of the Moyal product is the first way. In the second approach, one re-interprets all the fields as operators in the Hilbert space which provides for a representation of the fundamental algebra that defines the underlying non-commutative geometry. In this note we follow the latter approach and we will closely follow the notation of Polychronakos [19.

Specializing to the $2+1$ dimensional case, the non-commutative geometry with coordinates $X, Y$ and $t$, and the corresponding derivative operators (which act via the adjoint action) $\partial_{X}, \partial_{Y}$ and $\partial_{t}$ satisfy the algebra

$$
\begin{array}{r}
{[X, Y]=i \theta} \\
{\left[\partial_{X}, \partial_{Y}\right]=\frac{-i}{\theta}} \\
{\left[\partial_{X}, X\right]=\left[\partial_{Y}, Y\right]=1} \\
{\left[\partial_{X}, Y\right]=\left[\partial_{Y}, X\right]=0}
\end{array}
$$

and $t$ and $\partial_{t}$ commute with all of the above operators. We take $\theta>0$, the case $\theta<0$ is obtained via a parity reflection.

The algebra of the non-commutative geometry allows us to define annihilation and creation operators (note $\theta>0$ )

$$
\begin{aligned}
a & =\frac{-i}{\sqrt{2 \theta}}(X+i Y) \\
a^{\dagger} & =\frac{i}{\sqrt{2 \theta}}(X-i Y)
\end{aligned}
$$

Clearly $\left[a, a^{\dagger}\right]=1$. The usual Fock space basis states $\mid k>$ are obtained via the action 
of the annihilation and creation operators: $a\left|0>=0, a^{\dagger}\right| k>=\sqrt{k+1} \mid k+1>$ and $a|k>=\sqrt{k}| k-1>$.

\section{Action and Equations of Motion and Comments}

\subsection{Action and Equations of motion}

The action that will interest [19] us is written as

$$
S=\operatorname{Tr}\left(\frac{1}{4}\left[D_{\mu}, D_{\nu}\right]\left[D^{\mu}, D^{\nu}\right]+\frac{i}{3} \lambda D^{3}+\frac{1}{2}\left[D_{\mu}, \Phi\right]\left[D^{\mu}, \Phi\right]-e V(\Phi)\right) .
$$

Here $\operatorname{Tr}=\int d t t r, t r$ is the trace over the associated Hilbert (Fock) space, $\lambda$ and $e$ are coupling constants, the operators $D_{\mu}$ are defined as

$$
D_{\mu}=-i \partial_{\mu}+A_{\mu}
$$

and $D=d x^{\mu} D_{\mu}$ is the covariant exterior derivative operator. The operators $D_{\mu}$ and $\Phi$ are hermitean operators. We define the non-commutative magnetic field by

$$
\left[D_{X}, D_{Y}\right]=i B
$$

These covariant derivatives transform as

$$
D_{\mu} \rightarrow U D_{\mu} U^{\dagger}
$$

under local gauge transformations $U . U$ is a unitary Hilbert space operator, hence an element of $U(\infty)$ and in general a function of $t$. The equations of motion resulting from the action are

$$
\begin{gathered}
{\left[D_{\nu},\left[D^{\nu}, D_{\mu}\right]\right]+\left[\Phi,\left[D_{\mu}, \Phi\right]\right]=i \lambda \epsilon_{\mu \nu \lambda}\left[D^{\nu}, D^{\lambda}\right]} \\
{\left[D_{\mu},\left[D^{\mu}, \Phi\right]\right]+e V^{\prime}(\Phi)=0 .}
\end{gathered}
$$

\subsection{Comments on Non-Commutative Yang-Mills Theories, the Coefficient of the Chern-Simons Term and Gauge Invariance}

We wish to make three points. First, we have not concerned ourselves with the distinction made between $U(1)$ non-commutative gauge theory and that based on a non-abelian group, say $U(N)$, as was done for example in Polychronakos [19. The reason is that the $U(1)$ theory contains all possible non-abelian theories within it, in a very precise and exact way (See also [8], [19], [14]). The representation Hilbert

space given in for example [19] for the non-abelian case is the tensor product of a finite dimensional vector space taken say for the $U(N)$ factor with the standard 
infinite dimensional Hilbert (Fock) space for the representation of the algebra of the non-commutative geometry. Thus a basis of this space, $\mathcal{A}$, is for example given by

$$
\mathcal{A}=\{\mid a) \otimes \mid k>, \quad a \in 1 \cdots N, \quad k \in 0,1,2, \cdots\}
$$

and gauge transformations consist of unitary operators $V$ which act in this space. It is evident that a relabelling of these basis states can yield a basis which appears identical to the usual Fock basis without the $N \times N$ factor. For example if we define $\mathcal{B}$ such that

$$
\begin{aligned}
\mathcal{B} & =\{\mid b>>, \quad b=0,1,2, \cdots, \\
& \left.\ni|b>>=| a) \otimes \mid k>, \quad a=b \bmod N, \quad k=\frac{b-b \bmod N}{N},\right\}
\end{aligned}
$$

and the unitary transformations $V$ are simply unitary transformations on the Fock space relabelled as in the basis $\mathcal{B}$. Hence the non-commutative $S U(N)$ gauge theory is completely contained inside the so-called non-commutative $U(1)$ gauge theory. Perhaps a better name for this gauge theory would be the $U(\infty)$ gauge theory. We wish to make clear that there is still some utility to look at non-commutative YangMills theories in the tensor product picture, indeed, we will use it later. Our point is simple, that the $U(1)$ theory actually contains all possible gauge groups inside it.

Secondly, the coefficient of the Chern-Simons term (as written in equation (2.1)) is imaginary for the action in Minkowski space, hence $\lambda$ is real. This yields a real action for hermitean operators $D_{\mu}$. Analytic continuation to Euclidean space results in the appearance of a relative $i$ between the Chern-Simons term, which is odd in temporal derivatives, and the rest of the Lagrangian which is even. The ensuing Euclidean operator equations of motion have no solution in terms of hermitean operators $D_{\mu}$. This fact is familiar in the usual commuting case, where Euclidean, instanton solutions for theories containing Chern-Simons terms exist only for complex fields, ie. complex monopoles [20] or complex vortex strings [21]. Hence the hermitean solution by Polychronakos [19] for the Euclidean case is not valid for the usual Euclidean theory, however it is a solution of this somewhat different theory.

Our third, related point is that the Chern-Simons action is actually, at least formally, perfectly gauge invariant. Consequently the coefficient of the Chern-Simons term, for the Euclidean theory, need not even be imaginary, relative to the other terms in the action. Furthermore, the coefficient of the Chern-Simons term need not be quantized, as one must take in the case of a commutative non-abelian gauge theory [25]. Hence one can actually entertain a Euclidean action and equations of motion where $\lambda$ is real, with the solution in terms of hermitean operators as given by Polychronakos [19]. The analytic relationship of such a Euclidean theory and its solutions is not to the usual Minkowski theory with real Chern-Simons term, but one with imaginary coefficient $\lambda$. Obviously, such a theory has no counterpart in the usual, commutative theatre. 


\section{Solutions}

\subsection{Previously found solutions}

It is useful to rewrite the equations of motion in terms of the operators

$$
D=\frac{D_{X}+i D_{Y}}{\sqrt{2}}, \quad D^{\dagger}=\frac{D_{X}-i D_{Y}}{\sqrt{2}}
$$

which yields the equations

$$
\begin{gathered}
{\left[D,\left[D^{\dagger}, D_{0}\right]\right]+\left[D^{\dagger},\left[D, D_{0}\right]\right]-\left[\Phi,\left[D_{0}, \Phi\right]\right]=2 \lambda\left[D, D^{\dagger}\right]} \\
{\left[D_{0},\left[D_{0}, D\right]\right]+\left[D,\left[D, D^{\dagger}\right]\right]+[\Phi,[D, \Phi]]=2 \lambda\left[D_{0}, D\right]} \\
{\left[D_{0},\left[D_{0}, \Phi\right]\right]-\left[D,\left[D^{\dagger}, \Phi\right]\right]-\left[D^{\dagger},[D, \Phi]\right]+e V^{\prime}(\Phi)=0}
\end{gathered}
$$

It may be noted that whereas for $\lambda=e=0$, the field eqs. (3.2) to (3.4) are scale invariant, the scale invariance is lost in case $e=0$ but $\lambda \neq 0$. In particular, for $e=\lambda=0$ the field eqs. are unchanged under $\left(D, D_{0}, \Phi\right) \rightarrow \alpha\left(D, D_{0}, \Phi\right)$. In fact, they are also invariant under $(D, \Phi) \rightarrow \alpha(D, \Phi), D_{0} \rightarrow \pm \alpha D_{0}$.

The previously found flux tube solutions at $\lambda=0=e$ are obtained most efficiently via the solution generating technique of Harvey, Kraus and Larsen (HKL) [22], from the simple vacuum configuration

$$
D_{0}=-i \partial_{t}, \quad D=\sqrt{\frac{2}{\theta}} a, \quad \Phi=\Phi_{0}
$$

with $\Phi_{0}=\phi_{0} \mathbb{I}, \phi_{0}$ a $c$-number and $\mathbb{I}$ is the identity operator. Obviously the generalization, to the case $e \neq 0$ with a potential of the form, for example

$$
e V(\Phi)=e\left(\Phi^{2}-\Phi_{0}^{2}\right)^{2}
$$

still yields a solution. The flux tube solutions of Polychronakos and others are obtained, as enunciated by HKL [22] via the adjoint action with the operator

$$
S=\sum_{k=0}^{\infty}|k+1><k|
$$

which satisfies

$$
S S^{\dagger}=\hat{P}_{0}=\mathbb{I}-|0><0|
$$

while

$$
S^{\dagger} S=\mathbb{I I} .
$$

Then

$$
D_{\mu} \rightarrow S^{n} D_{\mu}\left(S^{\dagger}\right)^{n}
$$




$$
\Phi \rightarrow S^{n} \Phi\left(S^{\dagger}\right)^{n}
$$

yields a solution with a flux tube of $n$ units of magnetic flux.

A minor comment is in order concerning the HKL construction. It is not necessary that the states chosen to construct the operator $S$ be the usual states of the Fock basis, $\{\mid k>, \quad k=0,1,2, \cdots\}$, created by the action of $a^{\dagger}$ on the vacuum. Indeed, any orthonormal basis $\left\{\left|j>>=\Sigma_{k} \alpha_{j, k}\right| k>\right\}$ can be used to construct $S$. We will use this freedom later.

\subsection{A new solution}

We exhibit a new static solution, where the action of $D_{0}$ gives 0 , of the equations for $\lambda=0$ and for $e=0$. The choice above $\Phi=\Phi_{0}$ renders the commutators

$$
\left[D, \Phi_{0}\right]=\left[D^{\dagger}, \Phi_{0}\right]=0 .
$$

From the structure of the equations (3.2) to (3.4) it is clear that this is a sufficient condition but not a necessary one. Another sufficient condition for $e=0$ is given by

$$
[D, \Phi]=-\left(\left[D^{\dagger}, \Phi\right]\right)^{\dagger}=\alpha \mathbb{I}
$$

where $\alpha$ is an arbitrary $c$-number, since the equations of motion now only include commutators of these quantities with other operators. Hence we can take

$$
\begin{aligned}
D & =\sqrt{\frac{2}{\theta}} a \\
\Phi & =\alpha\left(a+a^{\dagger}\right)
\end{aligned}
$$

which yields another, new solution that is not obtainable from the vacuum solution (3.5) by the solution generating method of HKL. Of course we could act with the HKL method on this new solution to get more solutions:

$$
\begin{gathered}
D=\sqrt{\frac{2}{\theta}} S^{n} a\left(S^{\dagger}\right)^{n} \\
\Phi=\alpha S^{n}\left(a+a^{\dagger}\right)\left(S^{\dagger}\right)^{n}
\end{gathered}
$$

For $\lambda \neq 0$ we have not found the smooth, static extension of this solution, but for the previously found solutions we do have such extensions, to which we turn next.

\subsection{Solutions with Chern-Simons term}

It suffices to find the extension of the vacuum solution (3.5) to the case $\lambda \neq 0$ because of the power of the method of HKL. The solution can be exhibited in two ways, via a reasonably general hypothesis for the coefficients in a Fock space basis for the operators in question or derived as a relatively uncomplicated ansatz which is shown to satisfy the equations of motion. We will show both forms for the solution as they are instructive. The solution was originally found using a non-static temporal gauge description. We note that as in the commutative case, the Chern-Simons term induces a non-zero electric field whenever there is magnetic flux present. 


\subsubsection{Time dependent solutions}

One way of obtaining the solution is to consider the temporal gauge, $A_{0}=0$, so that $D_{0}=-i \partial_{t}$, and to look for a time dependent solution. The equations of motion become:

$$
\begin{gathered}
\ddot{D}-\left[D,\left[D, D^{\dagger}\right]\right]=2 i \lambda \dot{D}, \\
{\left[D, \dot{D}^{\dagger}\right]+\left[D^{\dagger}, \dot{D}\right]=-2 i \lambda\left[D, D^{\dagger}\right] .}
\end{gathered}
$$

We take $\Phi=\Phi_{0}$ so that it decouples from the equations. With the hypothesis

$$
\begin{aligned}
D & =f(N) a \\
& =\sum_{n=0}^{\infty} f_{n}|n><n| a=\sum_{n=0}^{\infty} f_{n} \sqrt{n+1}|n><n+1| \\
& \equiv \sum_{n=0}^{\infty} g_{n}|n><n+1|
\end{aligned}
$$

we find

$$
\begin{aligned}
& B=\left[D, D^{\dagger}\right]=\sum_{n=0}^{\infty}\left|g_{n}\right|^{2}(|n><n|-|n+1><n+1|), \\
& {[D, B]=\sum_{n=0}^{\infty} g_{n}\left(\left|g_{n+1}\right|^{2}-2\left|g_{n}\right|^{2}+\left|g_{n-1}\right|^{2}\right)(|n><n+1|,}
\end{aligned}
$$

with the definition $g_{-1}=0$. Hence the field eqs. (3.15) and (3.16) give:

$$
\begin{gathered}
\ddot{g}_{n}-g_{n}\left(\left|g_{n+1}\right|^{2}-2\left|g_{n}\right|^{2}+\left|g_{n-1}\right|^{2}\right)=2 i \lambda \dot{g}_{n} \\
\left(g_{n} \dot{g}_{n}^{*}-\dot{g}_{n} g_{n}^{*}\right)+2 i \lambda\left|g_{n}\right|^{2}=\left(g_{n-1} \dot{g}_{n-1}^{*}-\dot{g}_{n-1} g_{n-1}^{*}\right)+2 i \lambda\left|g_{n-1}\right|^{2} .
\end{gathered}
$$

Defining

$$
g_{n}=R_{n} e^{i \gamma_{n}}
$$

the eq. (3.21) simplifies to

$$
2 i R_{n}^{2}\left(-\dot{\gamma}_{n}+\lambda\right)-2 i R_{n-1}^{2}\left(-\dot{\gamma}_{n-1}+\lambda\right)=0 .
$$

which implies

$$
R_{n}^{2}\left(-\dot{\gamma}_{n}+\lambda\right)=0
$$

since $R_{-1}=0$. If $R_{n} \neq 0$ we get

$$
\dot{\gamma}_{n}=\lambda \quad \text { i.e. } \quad \gamma_{n}=\lambda t+\gamma_{n}(0),
$$

where $\gamma_{n}(0)$ allows for arbitrary constant phases for $g_{n}$, which we notationally suppress. Then the imaginary part of eq. (3.20) implies that $R_{n}$ is time independent and the real part yields

$$
R_{n}\left(R_{n+1}^{2}-2 R_{n}^{2}+R_{n-1}^{2}-\lambda^{2}\right)=0 .
$$


This equation (with $\lambda=0$ ) has been analyzed with clarity by Polychronakos. On applying his derivation to our case of $\lambda \neq 0$, we find that as in [19], it has solutions of the form $R_{-1}=R_{0}=\cdots=R_{N-1}=0$ and the remaining $R_{n} \neq 0, n \geq N$. We will take $N=0$ so that $R_{0} \neq 0$, the higher solutions are obtained via the method of HKL. The recurrence relation is easily solved

$$
R_{n}^{2}=\lambda^{2} \frac{n(n+1)}{2}+\left(R_{0}^{2}\right)(n+1)
$$

and $R_{0}^{2}=\frac{2}{\theta}$ by continuity with the solution at $\lambda=0$. This gives the solution, restoring the constant phases

$$
g_{n}=e^{i\left(\lambda t+\gamma_{n}(0)\right)} \sqrt{\lambda^{2} \frac{n}{2}+\frac{2}{\theta}} \sqrt{n+1},
$$

hence

$$
D=e^{i \lambda t} e^{i \gamma(N)} \sqrt{\lambda^{2} \frac{N}{2}+\frac{2}{\theta}} a
$$

and

$$
B=\left[D, D^{\dagger}\right]=\lambda^{2} N+\frac{2}{\theta} .
$$

We can make an minor elaboration on the solutions in the sector with flux $\mathrm{N}$ for $e=0$. Here the first $N g_{n}$ 's are zero, ie. $g_{-1}=g_{0}=\cdots=g_{n-1}=0$ and $g_{n} \neq 0$ for $n \geq N$. For such a solution the possible configuration for $\Phi$ can be slightly more complicated. Indeed the adjoint action of $S^{N}$ yields

$$
\Phi=\Phi_{0}\left(\mathbb{I}-\hat{P}_{N}\right)
$$

where

$$
\hat{P}_{N}=\sum_{n=0}^{N-1}|n><n| .
$$

Hence $\Phi$ is proportional to the projection operator that projects on to the complement of the sub-space spanned by the first $N$ basis vectors. However, the gauge field is trivial in the sub-space spanned by the first $N$ basis vectors, hence $\Phi$ can actually be an arbitrary operator in this subspace:

$$
\Phi=\hat{P}_{N} \Phi_{N} \hat{P}_{N}+\Phi_{0}\left(\mathbb{I I}-\hat{P}_{N}\right)
$$

where $\Phi_{N}$ is an absolutely arbitrary operator .

\subsubsection{Static Gauge Ansatz}

The other way of obtaining the solution is to consider static solutions. Further, we again take $\Phi=\Phi_{0}$ so that it decouples from the field equations and we only need to 
solve field eqs. (3.2) and (3.3). We now now notice that the field eq. (3.2) is trivially satisfied if

$$
\left[D_{0}, D\right]=\lambda D
$$

Further, eq. (3.3) now requires that

$$
\left[D,\left[D, D^{\dagger}\right]\right] \equiv[D, B]=\lambda^{2} D
$$

We thus find that all solutions of the two eqs. (3.34) and (3.35) (with $\Phi=\Phi_{0}$ ) will automatically satisfy the field eqs. (3.2) to (3.4). So the question boils down to finding the different solutions to the two eqs. (3.34) and (3.35). Clearly, there could be several solutions to these two equations. One simplest possibility is that

$$
\left[D, D^{\dagger}\right]=-\lambda D_{0}
$$

so that in view of eq. (3.34), eq. (3.35) is automatically satisfied. The existence of such solutions has been mentioned by Polychronakos 23 where he pointed out that representations of $S U(1,1)$ provide for solutions of the equations of motion. An illustration is obtained from the above time-dependent solution, by transforming to the static gauge. If we choose

$$
D_{0}=-\lambda N=-\lambda \sum_{n=0}^{\infty} n|n><n|,
$$

while $D$ is as given in the time-dependent case (suppressing constant phases), i.e.

$$
D=\sum_{n=0}^{\infty} R_{n}|n><n+1|,
$$

then eq. (3.34) is trivially satisfied while eq. (3.35) is satisfied provided $R_{n}$ satisfies eq. (3.26). Thus, we find that the static solution is given by

$$
\begin{aligned}
D_{0} & =-\lambda N \\
D & =e^{i \gamma(N)} \sqrt{\frac{\lambda^{2}}{2} N+\frac{2}{\theta}} a \\
\Phi & =\Phi_{0} .
\end{aligned}
$$

As expected, $B \equiv\left[D, D^{\dagger}\right]$ being a gauge invariant quantity, is as previously found in the time-dependent case and given by eq. (3.30). One might argue that $B \equiv$ $\left[D, D^{\dagger}\right] \neq-\lambda D_{0}$. However, if one redefines $D_{0}$ by

$$
D_{0}=-\lambda N-\frac{2}{\lambda \theta} \mathbb{I}
$$

we recover 3.36. However, it is possible that there could be solutions for which eq. (3.36) is not valid. 


\subsubsection{More General Time-Dependent Solution}

We can generalize the time-dependent solution given above by choosing a higher moment ansatz for $D$ as in 19

$$
D=f(N) a^{k}=\sum_{n=0}^{\infty} g(n)|n><n+k|,
$$

while still assuming $\Phi=\Phi_{0}$. On exactly following the algebra as given in eqs. (3.18) to (3.25), one finds that instead of eq. (3.26), now $R_{n}$ satisfies the equation

$$
R_{n}\left(R_{n+k}^{2}-2 R_{n}^{2}+R_{n-k}^{2}-\lambda^{2}\right)=0
$$

with $R_{-1}=R_{-2}=\ldots=0$. This equation decouples into $k$ equations, each involving the coefficients $R_{k n+q}$ for single $q=0,1,2, \ldots, k-1$. On assuming $R_{0,1, \ldots, k-1}$ to be non-zero, the solution to the set of eqs. (3.42) is easily obtained

$$
R_{n k+q}^{2}=\frac{n(n+1)}{2} \lambda^{2}+(n+1) R_{q}^{2}, \quad q=0,1, \ldots, k-1 .
$$

This solution comes about because the equations are universal and here are applied in the sense of equations with the gauge group $U(1)^{k}$. It would be interesting to find solutions with the full $U(k)$ involved.

\subsection{New Solution at $e=0$}

We can show the existence of new solutions at both $\lambda=0$ and $\lambda \neq 0$. So far as we are aware, these solutions, even at $\lambda=0$ were not known before. We start with the hypothesis

$$
\begin{aligned}
& \Phi=\sum_{m=0}^{\infty} r_{m}|m><m| \\
& D=\sum_{n=0}^{\infty} g_{n}|n><n+1|
\end{aligned}
$$

Then

$$
\begin{aligned}
{[D, \Phi] } & =\sum_{n=0}^{\infty} g_{n}\left(r_{n+1}-r_{n}\right)|n><n+1| \\
{[\Phi,[\Phi, D]] } & =\sum_{n=0}^{\infty} g_{n}\left(r_{n+1}-r_{n}\right)^{2}|n><n+1| \\
{\left[D^{\dagger},[D, \Phi]\right] } & =\sum_{n=0}^{\infty}\left|g_{n-1}\right|^{2}\left(r_{n}-r_{n-1}\right)-\left|g_{n}\right|^{2}\left(r_{n+1}-r_{n}\right)|n><n| .
\end{aligned}
$$

As a result, the equations of motion (3.2) to (3.4) take the form

$$
\ddot{g}_{n}-g_{n}\left(\left|g_{n+1}\right|^{2}-2\left|g_{n}\right|^{2}+\left|g_{n-1}\right|^{2}\right)-g_{n}\left(r_{n+1}-r_{n}\right)^{2}=2 i \lambda \dot{g}_{n},
$$




$$
\begin{gathered}
\left(g_{n} \dot{g}_{n}^{*}-\dot{g}_{n} g_{n}^{*}\right)+2 i \lambda\left|g_{n}\right|^{2}=\left(g_{n-1} \dot{g}_{n-1}^{*}-\dot{g}_{n-1} g_{n-1}^{*}\right)+2 i \lambda\left|g_{n-1}\right|^{2}, \\
-\ddot{r}_{n}-2\left(\left|g_{n-1}\right|^{2}\left(r_{n}-r_{n-1}\right)-\left|g_{n}\right|^{2}\left(r_{n+1}-r_{n}\right)\right)=0 .
\end{gathered}
$$

A solution to eqs. (3.47) and (3.48) is obtained with the hypothesis $\ddot{r}_{n}=0$ and $g_{n}=R_{n} e^{i \lambda t}$ which yields

$$
r_{n+1}-r_{n}=\frac{c}{\left|g_{n}\right|^{2}}
$$

where $c$ is an arbitrary constant. Consequently, eq. (3.46) takes the form

$$
R_{n}\left(R_{n+1}^{2}-2 R_{n}^{2}+R_{n-1}^{2}-\lambda^{2}-\frac{c^{2}}{R_{n}^{4}}\right)=0 .
$$

Consider the case $R_{-1}=0, R_{0} \neq 0$. Then we get the equation

$$
R_{n+1}^{2}-R_{n}^{2}-\left(R_{n}^{2}-R_{n-1}^{2}\right)=\lambda^{2}+\frac{c^{2}}{R_{n}^{4}}
$$

Summing twice, first for $n=0 \cdots N$ and then for $N=0 \cdots M$ yields the equation (analogous to an integral equation)

$$
R_{M+1}^{2}=\lambda^{2} \frac{(M+1)(M+2)}{2}+(M+2)\left(R_{0}^{2}\right)+c^{2} \sum_{N=0}^{M} \sum_{n=0}^{N} \frac{1}{R_{n}^{4}} .
$$

A self-consistent perturbative solution ensues for $R_{n}^{2}$ for small $c^{2}$. With $R_{0}^{2}=\frac{2}{\theta}$ we get $R_{n}^{2}=\frac{\lambda^{2} n(n+1)}{2}+\frac{2}{\theta}(n+1)$ for $c^{2}=0$, which is the same solution as found previously. Then we can generate a perturbative expansion in $c^{2}$ by simply iterating the equation, for example to first order in $c^{2}$ we have

$$
R_{M+1}^{2}=\lambda^{2} \frac{(M+1)(M+2)}{2}+\frac{2}{\theta}(M+2)+c^{2} \sum_{N=0}^{M} \sum_{n=0}^{N} \frac{1}{\left(\lambda^{2} \frac{n(n+1)}{2}+\frac{2}{\theta}(n+1)\right)^{2}} .
$$

The latter sum is convergent and finite as $M \rightarrow \infty$ and there is no obstruction to iterating this process to generate a well defined perturbative expansion for $R_{n}$.

This solution can be immediately generalized to the case of $U(1)^{k}$. We keep the same ansatz for $\Phi$ but for $D$ we assume

$$
D=\sum_{n=0}^{\infty} g(n)|n><n+k|
$$

On following exactly the same steps as given above, a solution is obtained with the hypothesis $\ddot{r}_{n}=0$ and $g_{n}=R_{n} e^{i \lambda t}$ which now yields

$$
r_{n+k}-r_{n}=\frac{c}{\left|g_{n}\right|^{2}}
$$


where $c$ is an arbitrary constant. Consequently, we now have

$$
R_{n}\left(R_{n+k}^{2}-2 R^{2}(n)+R_{n-k}^{2}-\lambda^{2}-\frac{c^{2}}{R_{n}^{4}}\right)=0 .
$$

We consider the case $R_{-1}=R_{2}=\ldots=0$, and $R_{0,1, \ldots, k-1} \neq 0$. Then we get the equation

$$
R_{n+k}^{2}-2 R_{n}^{2}+R_{n-k}^{2}=\lambda^{2}+\frac{c^{2}}{R_{n}^{4}} .
$$

This equation groups into $k$ uncoupled subsystems each involving the coefficients $R_{k n+q}$ for a single $(q=0,1,2, \ldots, k-1)$. We can solve each of these $k$ equations as above.

\section{A Re-Formulation of the Non-Commutative Abelian Higgs Model}

In closing we exhibit a re-formulation of the Abelian Higgs model, where usually the Higgs scalar is taken to be in the fundamental representation. This is complementary to the usual form taken for matter fields in non-commutative gauge theories where, typically the adjoint action is used when concerning the gauge transformation properties of the matter. It is easy to re-formulate matter in the fundamental representation in terms of real fields transforming under an adjoint action. This is quite familiar in the commutative case, indeed any complex representation can be re-formulated as a sub-group of a larger group acting on exclusively real fields via an adjoint action. It is somewhat more complicated in the non-commutative theatre, but everything does go through.

Up to now we have found flux tube solutions in the abelian $(U(1))$ non-commuta-tive gauge theory. These flux tubes do not resemble the usual, Nielsen-Olesen [6] vortex type solitons familiar in the commutative theatre. In the models considered so far, the Higgs field has essentially been a spectator, except for the one configuration where we could only obtain the solution implicitly in subsection (3.4). To recover the Nielsen-Olesen type configurations we have to consider the Higgs field in the fundamental representation. However, every fundamental representation corresponds to the adjoint action in an appropriate, higher dimensional representation acting on a real (hermitean) space. For example, the fundamental representation of $S U(2)$ exists inside $S O(4)$, the fundamental of $S U(3)$ exists inside $S O(6)$, etc. Indeed the fundamental representation of $U(1)$, ie. a complex field, can be written equivalently as two real fields transforming via an adjoint action in $S O(2)$.

Hence, consider the action, written exactly as before in eq. (2.1), with the same equations of motion as in (2.5) and (2.6). However, now the operators appearing in these equations are given by

$$
D_{\mu}=-i \partial_{\mu}+\sigma_{2} A_{\mu}
$$


while

$$
\Phi=\Phi_{1} \sigma_{1}+\Phi_{2} \sigma_{3}
$$

where $\sigma_{i}$ are the usual Pauli matrices. Hence we are using the nomenclature of two real fields, in two non-commutative dimensional $S O(2)$ Yang Mills theory. The $U(1)$ is explicitly extracted out of the $U(\infty)$ gauge symmetry of the usual non-commutative $U(1)$ gauge theory. Thus, although we find the notation redundant, it is still useful, as the equations of motion appear exactly as before. For $\lambda=0$ the vacuum solution is

$$
D_{0}=0, \quad D=\sqrt{\frac{2}{\theta}} a, \quad \Phi=\Phi_{0} \sigma_{1}
$$

$\Phi_{0}$ appearing in the potential is interpreted as before although now $\mathbb{I}=\mathbb{I}_{2 \times 2} \otimes$ $\mathbb{I}_{\text {Fock space }}$.

The vortex solution of Bak [16] can be obtained by the method of HKL with an appropriate choice for $S$. This choice can be found by comparing with Bak. Here

$$
\phi=\phi_{1}+i \phi_{2}=\frac{1}{\sqrt{N+1}} a^{\dagger}
$$

hence

$$
\begin{aligned}
& \phi_{1}=\frac{1}{2}\left(a \frac{1}{\sqrt{N+1}}+\frac{1}{\sqrt{N+1}} a^{\dagger}\right) \\
& \phi_{2}=\frac{1}{2 i}\left(a \frac{1}{\sqrt{N+1}}-\frac{1}{\sqrt{N+1}} a^{\dagger}\right)
\end{aligned}
$$

This expression for the field $\phi$ in Bak [16] is exactly the definition of the operator $S$ of HKL

$$
\phi=\sum_{n=0}^{\infty}|n+1><n| \equiv S
$$

hence

$$
\phi \phi^{\dagger}=S S^{\dagger}=\mathbb{I}_{\text {Fock space }}-|0><0|
$$

which is a projection operator. The construct $\Phi=\phi_{1} \sigma_{1}+\phi_{2} \sigma_{3}$ remarkably shares this property,

$$
\begin{aligned}
\Phi^{2} & =\phi_{1}^{2}+\phi_{2}^{2}+i \sigma_{2}\left[\phi_{1}, \phi_{2}\right] \\
& =\left(\frac{S+S^{\dagger}}{2}\right)^{2}+\left(\frac{S-S^{\dagger}}{2 i}\right)^{2}+i \sigma_{2}\left[\left(\frac{S+S^{\dagger}}{2}\right),\left(\frac{S-S^{\dagger}}{2 i}\right)\right] \\
& =\frac{1}{2}\left(S^{\dagger} S+S S^{\dagger}\right)+\frac{1}{2} \sigma_{2}\left(S^{\dagger} S-S S^{\dagger}\right) \\
& =\frac{1}{2}\left(2 \mathbb{I}-|0><0|-\sigma_{2}|0><0|\right)=\mathbb{I I}-\left(\frac{1+\sigma_{2}}{2}\right)|0><0|
\end{aligned}
$$

which is evidently a projection operator. We need to find the operator $\mathcal{S}$ such that

$$
\Phi=\Phi_{0}\left(\left(\frac{S+S^{\dagger}}{2}\right) \sigma_{1}+\left(\frac{S-S^{\dagger}}{2 i}\right) \sigma_{3}\right)=\mathcal{S} \Phi_{0} \sigma_{1} \mathcal{S}^{\dagger}
$$


It is not difficult to see that the solution for $\mathcal{S}$ is given by

$$
\mathcal{S}=\sigma_{+}+\sigma_{-} S
$$

where we choose to represent $\sigma_{ \pm}=\frac{1}{2}\left(\sigma_{1} \pm i \sigma_{3}\right)$. Then we find

$$
\begin{aligned}
\mathcal{S S}^{\dagger} & =\sigma_{+} \sigma_{-}+\sigma_{-} \sigma_{+} S S^{\dagger}=\sigma_{+} \sigma_{-}+\sigma_{-} \sigma_{+}\left(\mathbb{I}_{\text {Fock space }}-|0><0|\right) \\
& =\frac{1-\sigma_{2}}{2}+\frac{1+\sigma_{2}}{2}\left(\mathbb{I}_{\text {Fock space }}-|0><0|\right) \\
& =\mathbb{I}+\frac{1+\sigma_{2}}{2}|0><0| \equiv \mathbb{I}-\mathcal{P} \\
\mathcal{S}^{\dagger} \mathcal{S} & =\mathbb{I}
\end{aligned}
$$

where $\mathcal{P}$ is the appropriate projection operator, and

$$
\begin{aligned}
\mathcal{S} \Phi_{0} \sigma_{1} \mathcal{S}^{\dagger} & =\Phi_{0}\left(\sigma_{-} \sigma_{1} \sigma_{-} S+\sigma_{+} \sigma_{1} \sigma_{+} S^{\dagger}\right) \\
& =\Phi_{0}\left(\left(\frac{1+\sigma_{2}}{2}\right) \sigma_{1} S+\left(\frac{1-\sigma_{2}}{2}\right) \sigma_{1} S^{\dagger}\right) \\
& =\Phi_{0}\left(\left(\frac{S+S^{\dagger}}{2}\right) \sigma_{1}+\left(\frac{S-S^{\dagger}}{2 i}\right) \sigma_{3}\right)
\end{aligned}
$$

\section{Conclusions}

In this paper we have found the extensions of the flux tube type solitons in noncommutative Maxwell Higgs theories to the situtation where a Chern-Simons term is added, among other results. There are compelling reasons to consider such an extension. Indeed, non-commutative geometry violates parity at a fundamental level, the commutation relations between $X$ and $Y$ break parity. The resulting effective magnetic field also breaks parity. There is no reason to expect that the governing action should preserve it, and hence it is natural to consider the inclusion of the Chern-Simons term.

In addition, the non-commutative Chern-Simons theory is proposed to describe

the fractional quantum Hall effect [4] (see also [24]). The non-commutative pure Chern-Simons gauge theory is studied as a limiting low energy effective theory. A solution of the equations of motion was shown to describe the fractional quantum Hall fluid quasi-particles. The solution is conveniently expressed as

$$
\begin{aligned}
y_{1}-i y_{2} & =\sqrt{\frac{\theta}{2}} a \\
x_{1}-i x_{2} & =\sqrt{\frac{\theta}{2}} b
\end{aligned}
$$

where $b$ is the operator defined by

$$
b^{\dagger}|n>=\sqrt{n+1+\nu}| n+1>
$$




$$
\begin{aligned}
& b|n>=\sqrt{n+\nu}| n-1> \\
& b \mid 0>=0
\end{aligned}
$$

where $x_{i}$ are the coordinates of the fluid particles (Eulerian coordinates) while the $y_{i}$ are the co-moving coordinates of the fluid particles (Lagrangian coordinates) (see [1] for details) in the continuum limit and $\nu$ is the filling factor.

Our solution in the limit of large parameter $\lambda$ becomes

$$
D_{0}=-\lambda N, \quad D=\lambda \sqrt{\frac{N}{2}} a, \quad \Phi=\Phi_{0}
$$

which does not seem to reduce to the solution of Susskind 《4. It would be interesting to see the import of our solutions, especially with flux-tubes for the quantum Hall system. For example, the solution obtained from the above solution by conjugating with $S$ and $S^{\dagger}$ is

$$
D_{0}=-\lambda(N-\mathbb{I}+|0><0|), \quad D=\frac{\lambda}{\sqrt{2}} \sqrt{\frac{N(N-1)}{N+1}} a, \quad \Phi=\Phi_{0}(\mathbb{I}-|0><0|) .
$$

The energy of this configuration is given by

$$
E=\operatorname{tr}\left(\frac{1}{2}\left(B^{2}+\left[D^{\dagger}, D_{0}\right]\left[D_{0}, D\right]\right)+e V(\Phi)\right) .
$$

Normalizing so that the energy of the vacuum configuration, equation (5.3), is zero, we find that only the inhomogeneous potential contributes, taking for example $e V(\Phi)=e\left(\Phi^{2}-\Phi_{0}^{2}\right)^{2}$ gives

$$
E=\operatorname{tr}\left(e \Phi_{0}^{4}|0><0|\right)=e \phi_{0}^{4}
$$

\section{Acknowledgments}

We thank the the organizers of Strings 2001, Mumbai, where this paper germinated, for a superbly run, first class conference and for their seductive proselytization of ordinary field theorists to string theory. M.P. thanks the Institute of Physics, Bhubaneswar, for kind hospitality, where essentially all of this work was done. We

thank A. P. Polychronakos for useful correspondence. We also thank NSERC of Canada for financial support.

\section{References}

[1] A. Connes, M. Douglas and A. Schwartz, J. High Energy Phys. 9802 (1998) 003.

[2] M.R. Douglas and C. Hull, J. High Energy Phys. 9802 (1998) 008.

[3] N. Seiberg and E. Witten, J. High Energy Phys. 9909 (1999) 032. 
[4] L. Susskind, hep-th/0101029.

[5] T. Yoneya, hep-th/0004074.

[6] N. K. Nielsen and P. Olesen, Nucl. Phys. B 61 (1973) 45.

[7] R. Gopakumar, S. Minwalla and A. Strominger, J. High Energy Phys. 0005 (2000) 020 .

[8] D.J. Gross and N.A. Nekrasov, J. High Energy Phys. 0007 (2000) 034, J. High Energy Phys. 0010 (2000) 020, hep-th/0010090.

[9] K. Hashimoto, J. High Energy Phys. 0012 (2000) 023, hep-th/0010251.

[10] A. Hashimoto and K. Hashimoto, J. High Energy Phys. 9911 (1999) 005.

[11] M. Hamanaka and S. Terashima, hep-th/0010221.

[12] A.S. Gorsky, Y.M. Makeenko and K.G. Selivanov, Phys. Lett. B 492 (2000) 344.

[13] M. Aganagic, R. Gopakumar, S. Minwalla and A. Strominger,hep-th/0009142.

[14] D. Bak, K. Lee and J-H. Park, hep-th/0011099.

[15] G.S. Lozano, E.F. Moreno and F.A. Schaposnik, hep-th/0012266.

[16] D. Bak, hep-th/0008204.

[17] D.P. Jatkar, G. Mandal and S.R. Wadia, J. High Energy Phys. 0009 (2000) 018.

[18] G.S. Lozano, E.F. Moreno and F.A. Schaposnik, hep-th/0011205.

[19] A.P. Polychronakos, Phys. Lett. B 495 (2000) 407.

[20] B. Tekin, K. Sararian and Y. Hosotani, Nucl. Phys. B 539 (1999) 720.

[21] P. W. Irwin and M.B. Paranjape, hep-th/9906021.

[22] J.A. Harvey, P. Kraus and F. Larsen, hep-th/0010060.

[23] A.P. Polychronakos, J. High Energy Phys. 0011 (008) 2000.

[24] A.H. Chamseddine, J. Frohlich J. Math. Phys. 35 (1994) 5195; V. John, A. V. Nguyen, K. C. Wali, Phys. Lett. B 371 (1996) 252; C.-S. Chu, Nucl. Phys. B 580 (2000) 352; J. Kluson, hep-th/0012184; J. H. Brodie, hep-th/0012068; G.H. Chen, Y.-S. Wu, Nucl. Phys. B 593 (2001) 562; S. Gubser and R. Mukund, hep-th/0012155; S. Mukhi, N. V. Suryanarayana, J. High Energy Phys. 0011 (2000) 006; S. Gubser and S. Sondhi, hep-th/0006119; N. Grandi, G.A. Silva, hep-th/0010113; A.A. Bichl, J.M. Grimstrup, V. Putz, M. Schweda, J. High Energy Phys. 0007 (2000) 046.

[25] S. Deser, R. W. Jackiw, S. Templeton, Ann. Phys. (NY) 140 (1982) 372, ibid. 185 (1988) 406, ibid. 281 (2000) 409. 